\title{
An Update on Natural Occurrence and Biological Activity of Benzofurans
}

\section{Kavita Khatana* and Anjali Gupta}

Department of Chemistry, School of Basic and Applied Sciences, Galgotias University, Greater Noida, UP, India

*Corresponding Author: Kavita Khatana, Department of Chemistry, School of Basic and Applied Sciences, Galgotias University, Greater Noida, UP, India.
Received: June 28, 2020

Published: September 21, 2020

(C) All rights are reserved by Kavita Khatana.

\section{Graphical Abstract}

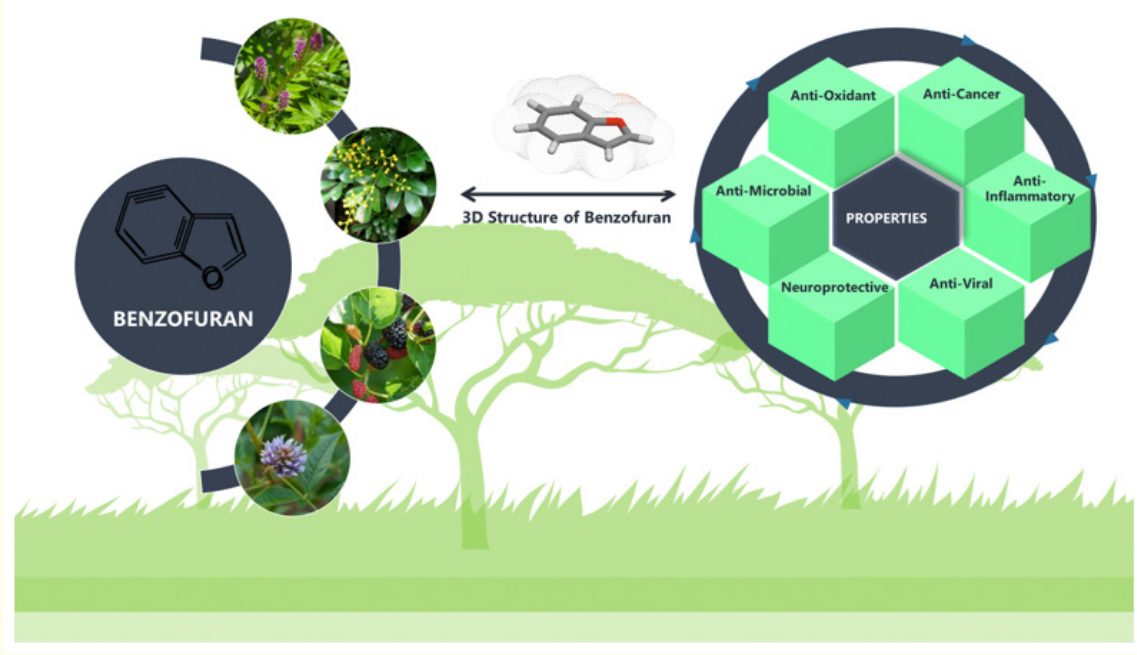

Figure a

\section{Abstract}

Benzofuran and its derivatives are the major group amongst the natural collection of biologically active heterocyclic compounds. Their wide range of pharmacological activities and imaginable applications in medicinal and pharmaceutical chemistry have attracted various research scholars, medicinal chemists and pharmacologists. This review emphasizes the progress and development of benzofuran derivatives in various biological activities with an update of current research findings during a decade.

Keywords: Benzofuran; Antioxidant; Antiviral; Antimicrobial; Anticancer

\section{Introduction}

Heterocyclic ring systems have emerged as powerful scaffolds for many biological evaluations. Heterocyclic compounds have significant role in medicinal and pharmacological chemistry, physiological and industrial world. In reference to pharmaceutical industry, over $60 \%$ of the top retailing drugs contain at least one heterocyclic motif as a part of overall topography of the compound. Benzofurans and its derivatives are important class of heterocyclic 
compounds, which are known to possess various biological properties. Benzofuran derivatives are versatile biodynamic agents that can be used to design and develop potentially useful therapeutic agents [1]. There is a great importance of several derivatives of furans in flavors and fragrances also. For example, furaneol is responsible for odor in strawberries; rose flower has odor due to rosefuran [3-methyl-2-(3-methylbut-2-enyl)-furan]; coffee shows its characteristics due to furylmethanethiol (Figure 1). Various natural benzofurans derivatives are also found to act as cytotoxic, anti-oxidant, anti-inflammatory, anti-fungal, neuroprotective and analgesic activity, anti-viral, edible, anti-allergic etc. A wide range of benzofurans have been isolated from natural sources. Their occurrence is confined to botanical species, zoological species and fungi species like, Aglaia perviridis, Eupatorium chinense, Tephrosia purpurea, Morus alba, Morus macroura, Liriope spicatavar. Prolifera, Eupatorium coelestinum, Ligularia veitchiana, Paeonia veitchii, Itea ilicifolia etc. Since many years, isolated benzofurans and their derivatives have been used for various biological activities e.g., anti-oxidants, antitumor, anti-inflammatory, anti-malarial, antiviral, exert anti-angiogenic effects (avoids the growth of blood vessels) etc. Numerous botanical species containing benzofuran derivatives are being used for treatment of headache, dizziness, anti-cancer drugs, anti-malarial drugs, migraine, epilepsy, infantile convulsion, tetanus etc.

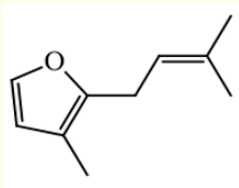

Rosefuran

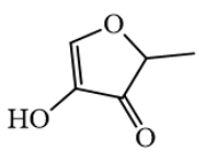

Furaneol

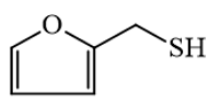

Furylmethanethiol
Figure 1

\section{Anti-cancer activity}

Benzofurans and its derivatives play an important role in treating cancerous cells, for example, benzofuran derivative, aglaodoratin (1), isolated from leaves of "Aglaia odorata" demonstrated the inhibition of HepG2 liver carcinoma cell proliferation via G2/M arrest and induced apoptotic cell death at a concentration of 25 $\mu \mathrm{M}$ [2]. Furthermore, aglapervirisin (2) isolated from leaves of 'Aglaia perviridis' was also known to possess significant cytotoxicity against human tumors cell lines [3]. Another research also revealed the cytotoxic potential of benzofuran analogs (3-12) extracted from seeds of Crataegus pinnatifida against HT-1080 human cancer cell lines. (E)-3-((2S,3S)-2-(4-hydroxy-3-methoxyphenyl)- 3-(hydroxymethyl)-7-methoxy-2,3-dihydrobenzo furan-5-yl) acryl aldehyde (12) displayed exceptional inhibitory activity (IC ${ }_{50}$ $=8.86 \mu \mathrm{M}$ ) as compared with positive control 5-fluorouracil (IC I0 $_{50}$ $=35.62 \mu \mathrm{M}$ ) [4]. Hang., et al. also studied the anticancer activity of $5 \alpha, 8 \alpha$-epoxy- $4 \alpha, 6 \beta$-dihydroxyamorphan-2-one (13) extracted from aerial parts of Eupatorium coelestinum which was found to strongly inhibit the activation of NF- $\kappa \mathrm{B}$ in TNF- $\alpha$-stimulated HeLa cells (IC ${ }_{50}$ of $12.4 \mu \mathrm{M}$ ) [5]. In a different study, two new benzofuran derivatives, 2-(4'-hydroxybenzyl)-5,6-methylenedioxy-benzofuran (14) and 2-(4'-hydroxybenzoyl)-5,6-methylenedioxybenzofuran (15), isolated from roots of "Liriope spicatavar. Prolifera" exhibited significant inhibitory activity against neutrophil respiratory burst with $\mathrm{IC}_{50}$ values $4.15 \pm 0.07$ and $5.96 \pm 0.37 \mu \mathrm{M}$, respectively [6]. Another series of compound 2-(3'-hydroxy-5'-methoxyphenyl)-3hydroxylmethyl-7-methoxy-2,3-dihydrobenzo furan-5-carboxylic acid (16) and 7-hydroxy-5,4'-dimethoxy-2-arylbenzofuran (17) which were isolated from the fruits of Livistona chinensis revealed potential anticancer activity against K562 and HL-60 human myeloid leukemia, CNE-1 human nasopharyngeal carcinoma cell lines and HepG2 human liver cancer with $\mathrm{IC}_{50}$ value ranging 5-150 $\mu \mathrm{M}$ [7]. 3-hydroxy-2-(3-methyl-2-butenyl)-1-(2-phenylethyl) benzofuran (18) isolated from twigs of "Macaranga kurzii" exhibited modest cytotoxicity against Hep G2 cell line with IC50 value of 30.14 $\mu \mathrm{g} / \mathrm{mL}$ [8]. Compounds 19-27 extracted from leaves of Morus notabilis were also found to show inhibition against PTP1B phosphatase activity in vitro [9]. Furthermore, a new benzofuran derivative 1-[6-hydroxy-2-isopropenyl-1-benzofuran-5yl]-1-ethanone (28) isolated from Petasites hybridus roots showed moderate inhibitory activity on human breast cancer MCF-7 cells proliferation in vitro with IC $_{50}$ value of $58 \mu$ mole/litre [10]. In another report, two novel chlorinated benzofurans, 3-hydroxy-1-(4,6,8-trichloro-1,9-dihydroxy-3,7-dimethoxydibenzo[b,d]furan-2-yl)propan-1-one (29) and 1-(4,6,8-trichloro-9-hydroxy-1,3,7-trimethoxydibenzo[b,d] furan-2-yl)butan-1-one (30) isolated from fruiting body of slime moulds of Polysphondylium filamentosum showed inhibitory activities on cell proliferation in mammalian cells and gene expression in Drosophila melanogaster [11]. 4,7,8-trimethoxy-2,3methylenedioxydibenzofuran (31), 7-hydroxy-4,8-dimethoxy-2,3methylene dioxydibenzofuran (32) isolated from aerial parts of Ribes takare showed mild $\alpha$-glucosidase inhibitory activity [12]. (E)-3-(2-(benzo[d][1,3] dioxol-5-yl)-7-methoxybenzofuran-5-yl) acrylaldehyde (33), a known benzofuran derivative, which is isolated from stem bark of "Duabanga grandiflora" used as cytotoxic activities against cancer cell line Acute Lymphoblastic Leukemia (MOLT-3) with $\mathrm{IC}_{50}$ value of $18.1 \mu \mathrm{M}$ [13]. Ganodone (34) extracted from the fruits of "Ganoderma tsugae" is used as a potential 
source of new anti-cancer medicines [14]. A known dihydrobenzofuran, 4-((2S,3R)-3-(hydroxymethyl)-5-(3-hydroxypropyl)7-methoxy-2,3-dihydrobenzofuran-2-yl)-2,6-dimethoxyphenol (35), extracted from Selaginella moellendorffii showed better inhibitory activity as compared with tirofiban against collagen induced aggregation of platelets [15]. In another report, compounds (E)-3-(2-(benzo[d][1,3]dioxol-5-yl)-7-methoxybenzofuran-5-yl)prop-2-en-1-ol (36) and (E)-methyl 3-(2-(benzo[d] [1,3]dioxol-5-yl)-7-methoxybenzofuran-5-yl)acrylate (37), isolated from the methnolic extract of the African medicinal plant "Zanthoxylum capens" also showed strong anticancer activity in HCT116 colon carcinoma cells [16]. A novel compound, 2,3-dihydro-2-[4-( $\beta$-glucopyranosyl $(1 \rightarrow 2)$-[ $\beta$-glucopyranosyl $(1 \rightarrow$ 6)]- $\beta$-glucopyranosyloxy)-3-methoxyphenyl]-3-(hydroxymethyl)7-methoxy-5-benzofuranpropanol (38) and a known compound (2R,3S,4S,5R,6S)-2-(hydroxymethyl)-6-(4-((2R,3S)-3(hydroxymethyl)-5-((E)-3-hydroxyprop-1-en-1-yl)-7-methoxy2,3-dihydrobenzofuran-2-yl)-2-methoxy phenoxy) tetrahydro-2Hpyran-3,4,5-triol (39) extracted from the plant of Castanea henryi acts as a key enzyme that is tyrosinase inhibitor [17]. Two compounds (40) and (41) isolated from the heartwood of D.odorifera. compound (40) showed positive results against myelogenous leukemia cell line K562, human hepatocellular carcinoma cell line BEL-7402 and human gastric carcinoma cell line SGC-7901. Compound (41) revealed the IC50 value of $33.5 \mu \mathrm{M}$ against BEL-7402 cell line for positive control paclitaxel [18]. A novel compound, 2,2-dimethyl-4-(3-methylbut-3-en-1-yn-1-yl)-2,3-dihydrobenzofuran-7-ol (42) extracted from Pestalotiopsis fici showed cytotoxic activity [19]. A known benzofuran, flavonolignan silybin (43) extracted from Silybum marianum showed anticancer properties [20].


$2 \mathrm{R}=\mathrm{OH} / \mathrm{OAC} ; 5^{\prime \prime \prime} \mathrm{R} / 5^{\prime \prime} \mathrm{S}$

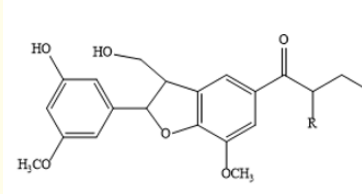

$3 \mathrm{R}=\mathrm{H} ; 7 \mathrm{~S}, 8 \mathrm{R} \quad 4 \mathrm{R}=\mathrm{OH} ; 7 \mathrm{R}, 8 \mathrm{~S}$

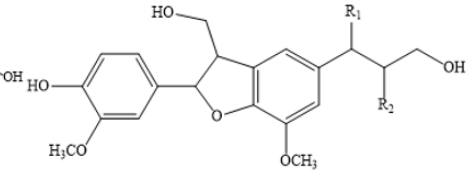

$5 \mathrm{R}_{1}=\mathrm{OCH}_{3}: \mathrm{R}_{2}=\mathrm{OH} ; 7 \mathrm{~S}, 8 \mathrm{R} ; 7^{\prime}, 8^{\prime}=$ Threol $6 \mathrm{R}_{1}=\mathrm{OCH}_{3} ; \mathrm{R}_{2}=\mathrm{OH} ; 7 \mathrm{R}, 8 \mathrm{8} ; 7^{\prime}, 8^{\prime}=$ Threo $7 \mathrm{R}_{1}=\mathrm{OCH}_{2} \mathrm{CH}_{3} ; \mathrm{R}_{2}=\mathrm{OH} ; 7 \mathrm{~S}, 8 \mathrm{R} ; 7^{\prime}, 8^{\prime}=$ Threo $8 \mathrm{R}_{1}=\mathrm{OCH}_{2} \mathrm{CH}_{3} ; \mathrm{R}_{2}=\mathrm{OH} ; 7 \mathrm{R}, 8 \mathrm{~S} ; 7^{\prime}, 8^{\prime}=$ Threo $9 \mathrm{R}_{1}=\mathrm{OCH}_{2} \mathrm{CH}_{3} ; \mathrm{R} 2=\mathrm{H} ; 7 \mathrm{R}, 8 \mathrm{~S}$<smiles>[Y13]C(CO)C([Y1])c1cc(OC)c2c(c1)[C@@H](CO)[C@H](c1ccc(O)c(OC)c1)O2</smiles>

$10 \quad 11 \mathrm{R}_{1}=\mathrm{H} ; \mathrm{R}_{2}=\mathrm{H} ; 7 \mathrm{R}, 8 \mathrm{~S}$

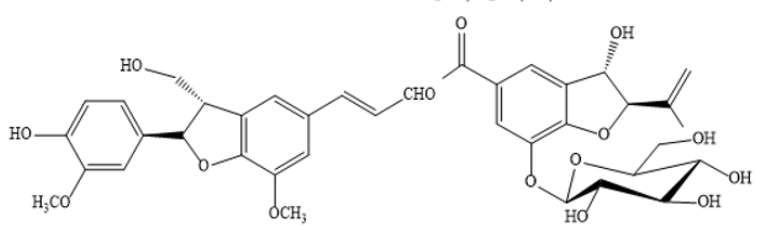

12<smiles>O=C(c1ccc(O)cc1)c1cc2ccc3c(c2o1)OCO3</smiles>

14

15<smiles>COc1ccc(-c2cc3cc(OC)cc(O)c3o2)cc1</smiles>

17<smiles>CC(C)=CCc1c(O)cc2occc2c1CCc1ccccc1</smiles>

18

19

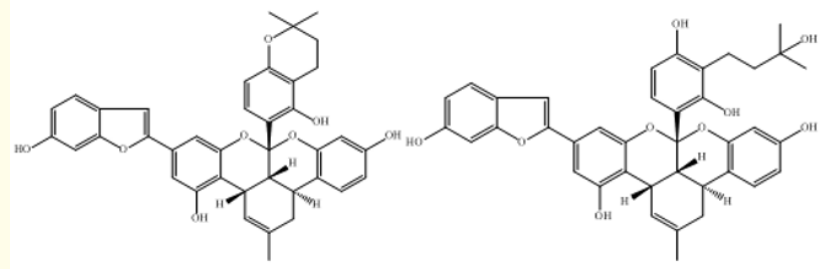

20

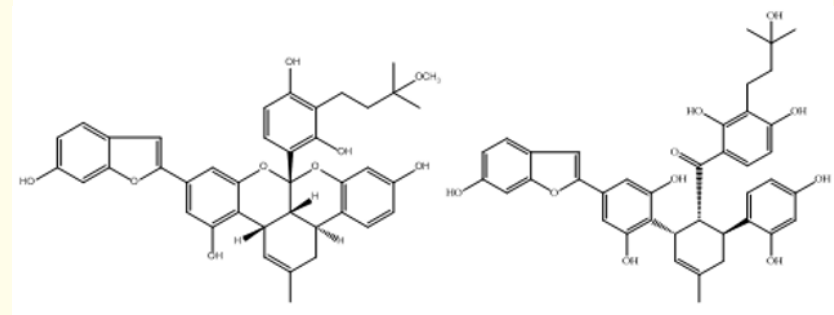

22

Citation: Kavita Khatana and Anjali Gupta. "An Update on Natural Occurrence and Biological Activity of Benzofurans". Acta Scientific Medical Sciences 4.10 (2020): 114-123. 


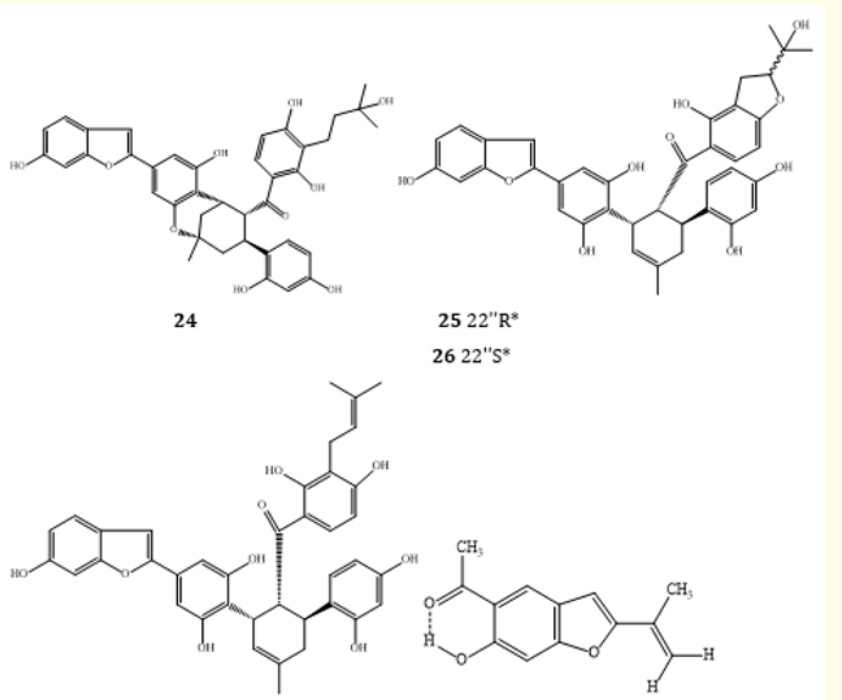

27

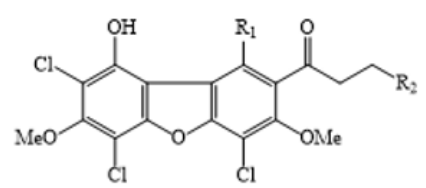

$29 \mathrm{R}_{1} / \mathrm{R}_{2}=\mathrm{OH} 30 \mathrm{R}_{1}=\mathrm{OMe}, \mathrm{R}_{2}=\mathrm{CH}_{3}$<smiles>COc1cc(/C=C/C=O)cc2cc(-c3ccc4c(c3)OCO4)oc12</smiles>



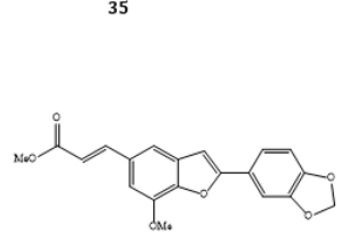

37

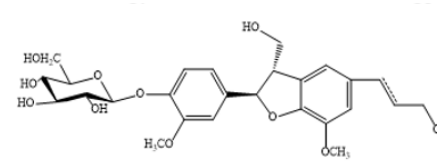

39

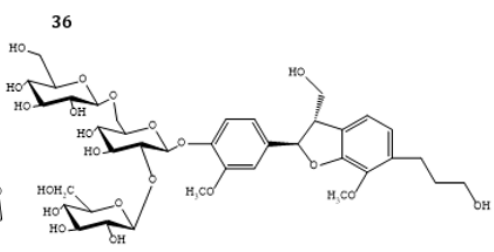

38

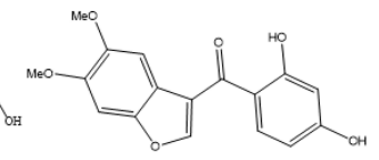

40

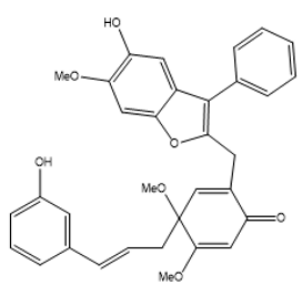

41

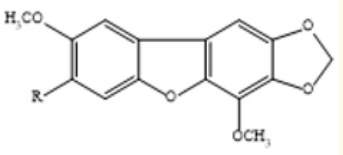

$31 \mathrm{R}=\mathrm{OCH}_{3} \quad 32 \mathrm{R}=\mathrm{OH}$

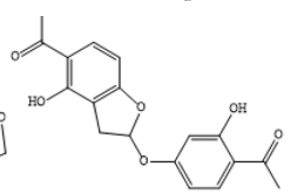

34<smiles>COc1cc(I)cc2cc(-c3ccc4c(c3)OCO4)oc12</smiles>

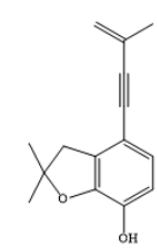

42

Figure 2

Anti-oxidant activity

Anti-oxidants act as defense system in our body. They protect us from heart disease, cancer, Alzheimer's, Parkinson's and cataract disease, etc. The compounds that can donate electrons and counteract free radicals have anti-oxidant properties. Anti-oxidant compounds can "donate" electrons to unstable free radicals so they don't have to snatch electrons from unsuspecting nearby cells. Antioxidants help in repairing of cells which are damaged by free radicals or we can say that they are taken as remedies to overcome the lethal action of oxygen free radicals. For example, a citation revealed significant antioxidant properties of compounds, 2-(2'-methoxy-4' hydroxy)-aryl-3-methy-6-hydroxy-benzofuran (44) and (Z)-2-(3,4dihydroxybenzylidene)-6-hydroxybenzofuran-3(2H)-one (45) extracted from Glycyrrhiza yunnanensis [21]. Two novel compounds, (E)-2-(3,4-dihydroxyphenyl)-3-formyl-4-hydroxy-5-(prop-1-en1-yl)benzofuran (46), iteafuranal A) and (E)-2-(3-hydroxy-4methoxyphenyl)-3-formyl-4-hydroxy-5-(prop-1-en-1-yl)benzofuran (47), iteafuranal B) extracted from aerial parts of "Itea ilicifolia" showed DPPH free radical scavenging capacity with an $\mathrm{IC}_{50}$ value of $0.34 \mathrm{mg} / \mathrm{mL}$ [22]. These Compounds showed ABTS cation radical scavenging capacity with $\mathrm{IC}_{50}$ values of 0.23 and $0.30 \mathrm{mg} / \mathrm{mL}$, respectively. Two new compounds, (7R,8S)-3,5'-dimethoxy-4',7-epoxy-8,3'-neolignane-5,9,9'-triol (48) and (2R)-12-hydroxy-4-methoxy-tremeton (49) isolated from aerial parts of "Leontopodium leontopodioides" were found to inhibit nitric oxide (NO) production in lipopolysaccharide-activated RAW264.7 macrophages with $\mathrm{IC}_{50}$ values of 35.80 and $24.41 \mu \mathrm{M}$, respectively [23]. Benzofuran derivatives 1-(4,6-dihydroxy-2-((1R,2S)1,2,3-trihydroxy-1-(2,4,6trihydroxy-5-(3-methyl butanoyl)cyclohexa-1,3-dien-1-yl) propyl)-5,6-dihydrobenzofuran-7-yl)-3-methylbutan-1-one (50) and 3-methyl-1-((2S,3'S,4'R,5'R)-3',4,4',5',6-pentahydroxy3a,3',4',5',6',7a-hexahydro-3H-spiro[benzofuran-2,2'-pyran]-7-yl) butan-1-one (51) obtained from roots of Lysidice rhodostegia exhibited potent antioxidative activity with IC $_{50}$ values of 3.29 and $3.39 \mu \mathrm{M}$, respectively [24]. 2-arylbenzofuran (52) extracted from root bark, stem bark and leaves of Morus alba reduced the oxidation of low-density lipoprotein (LDL's) responsible for atherogenesis [25]. Three novel benzofuran derivatives, i.e., $(2 R)-(4-$ methoxybenzyl)-5,7-dimethyl-6-hydroxyl-2,3-dihydrobenzofuran (53), 2-(2-hydroxyl-4-methoxy-benzyl)-5-methyl-6-methoxyl-2,3 dihydrobenzofuran (54), and 2-(4-hydroxy-benzyl)-5,6 dihydroxybenzofuran (55) and two known compounds 2-(4-methoxy-benzyl)-6,7-dimethoxyl-2,3-dihydrobenzofuran (56) and 2-(4-methoxybenzyl)-6,7-methylenedioxy-2,3-dihydrobenzofuran (57) extracted from the tubers of Ophiopogon japonicus revealed the effects on production of NO induced by LPS (lipopolysaccharide) in RAW264.7 cells [26]. Furthermore, two novel 2-arylben- 
zofuran dimers, shandougenines A (58) and shandougenines B (59) and six known benzofuran compounds, i.e., (60-65) isolated from ethanolic extract of Sophora tonkinensis, showed moderate to stronger DPPH free radical scavenging ability [27].

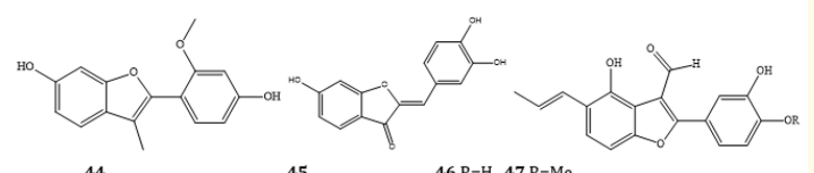<smiles>COc1cc(O)cc(C2Oc3c(O)cc(CCCO)cc3C2C[18O])c1</smiles>

48<smiles>C=C(C)C1Cc2c(ccc(C(C)=O)c2O)O1</smiles>

49<smiles>CC(C)CC(=O)C1=c2oc(C(c3c(O)cc(O)c(C(=O)CC(C)C)c3O)[C@H](O)CO)cc2=C([Hg])CC1O</smiles>

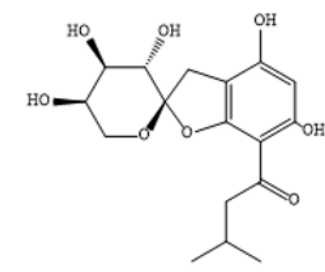

51

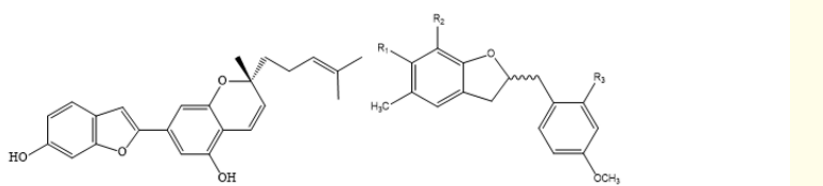

52

$53 \mathrm{R}_{1}=\mathrm{OH}, \mathrm{R}_{2}=\mathrm{CH}_{3}, \mathrm{R}_{3}=\mathrm{H}$
<smiles>[Y]c1cc(C2CC(=O)c3ccc(O)c([3H])c3O2)cc2c1OC(C(C)(C)C)C2</smiles>

$60 \mathrm{R}_{1}=\mathrm{R}_{2}=$ Isoprenyl

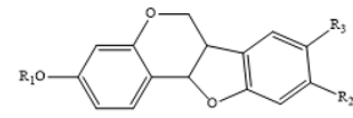

$61 \mathrm{R}_{1}=\mathrm{H}, \mathrm{R}_{2}+\mathrm{R}_{3}=-\mathrm{OCH}_{2} \mathrm{O}$ $62 \mathrm{R}_{1}=\mathrm{Gld}, \mathrm{R}_{2}+\mathrm{R}_{3}=-\mathrm{OCH}_{2} \mathrm{O}$ $63 \mathrm{R}_{1}=\mathrm{R}_{2}=\mathrm{OH}, \mathrm{R}_{3}=\mathrm{H}$

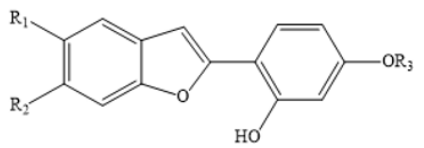

$64 \mathrm{R}_{1}=\mathrm{H}, \mathrm{R}_{2}=\mathrm{OH}, \mathrm{R}_{3}=\mathrm{CH}_{3}$

$65 \mathrm{R}_{2}=\mathrm{R}_{3}=-\mathrm{OCH}_{2} \mathrm{O}-, \mathrm{R}_{3}=\mathrm{H}$

\section{Anti-inflammatory-activity}

Anti-inflammatory is a characteristic/property of a substance which decreases the effect of pain, fever, tenderness, or inflammation. Many benzofurans and their derivatives which are extracted from plant, animal, fungus species showed anti-inflammatory activities. Novel compounds 6-methoxy-3-methyl-2-phenylbenzofuran5-ol (66) and 6-methoxy-2-methyl-3-phenylbenzofuran-5-ol (67) isolated from the heartwood of Dalbergia odorifera were shown to have protective effects against glutamate-induced oxidative injury in HT22 cells. These compounds were used in modulating the regulation of anti-inflammatory activity through the upregulation of heme oxygenase (HO)-1 in BV2 microglia [28]. Compound (68) isolated from the heartwood of D.odorifera inhibited the levels of proinflammatory mediators NO, PGE2, TNF- $\alpha$ and IL-1 $\beta$ with the results of decreased iNOS and COX-2 appearing when BV2 microglia were stimulated by LPS at the dose of $1 \mu \mathrm{g} / \mathrm{mL}$. 3-(2-hydroxy4-methoxyphenyl)benzofuran-6-ol (69) acted as a specific inhibitor of 5-lipoxygenase with an $\mathrm{IC}_{50}$ value of $0.08 \mu \mathrm{M}$ against the soluble rat enzyme; however, it was inactive against cyclooxygenase. 6-methoxy-3-phenylbenzofuran-5-ol (70), displayed protective effects with $\mathrm{EC}_{50}$ value of $(3.09 \mu \mathrm{M})$ [18]. Two novel compounds, (S)-1-((2S,3R)-2-(benzo[d][1,3] dioxol-5-yl)-3-(hydroxymethyl)7-methoxy-2,3-dihydrobenzofuran-5-yl)propane-1,3-diol (S)-3-((2S,3R)-2-(benzo[d][1,3 ]dioxol-5-yl)-3-(hydroxymethyl)7-methoxy-2,3-dihydrobenzofuran-5-yl)-3-methoxypropan-1-ol (72) showed anti-inflammatory activity, which is isolated from Breynia fruticosa [29]. A known compound (73), isolated from Breynia fruticose showed anti-inflammatory activity [29]. Two known compounds (74) and (75) isolated from Paeonia veitchii showed anti-inflammatory activity [30].
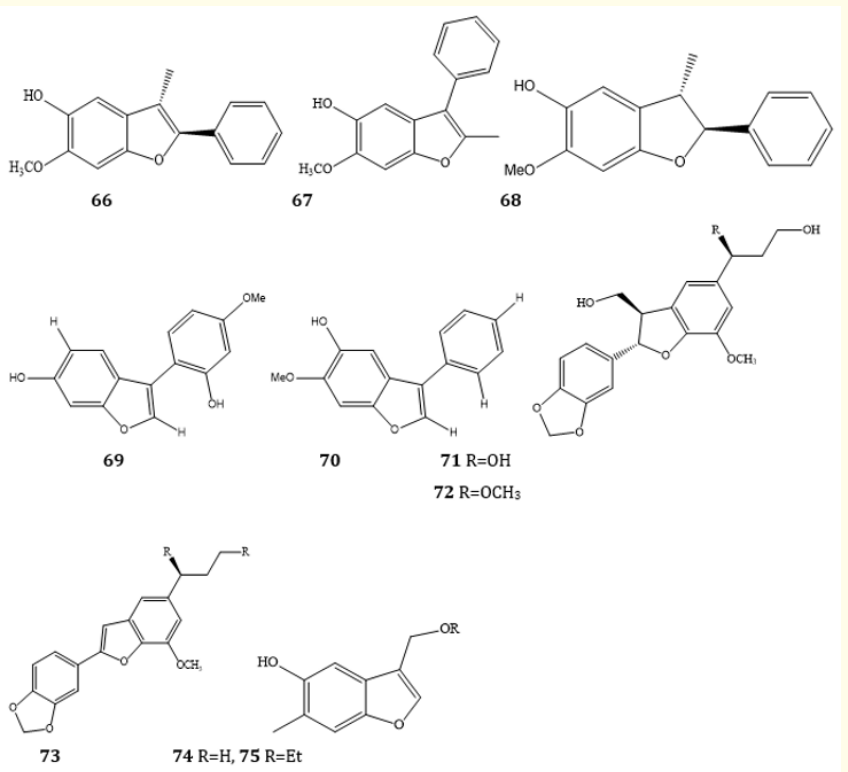

Figure 4

Figure 3 
Anti-microbial activities

Seven new compounds, i.e., 1-(2-((1S,4R)-7-acetyl-4,8-dihydroxy-1,4-bis(hydroxymethyl)-1,2,3,4-tetrahydrodibenzo[b,d] furan-1-yl)-5-hydroxybenzofuran-6-yl)ethanone 1-(2-((1S,4S)-7-acetyl-4,8-dihydroxy-1,4-bis(hyd roxymethyl)1,2,3,4-tetrahydrodibenzo[b,d]furan-1-yl)-5-hydroxybenzofuran6-yl)ethanone (77), (E)-1,1'-(2,2'-(4-methylpent-2-ene-2,4-diyl) bis(6-hydroxybenzofuran-5,2-diyl))diethanone (78), (E)-1,1',1"(2,2',2"-(2,6-dimethylhept-3-ene-2,4,6-triyl)tris(6-hydroxybenzofuran-5,2-diyl))triethanone (79), 1-(2-((1S,4R)-8-acetyl-4,7dihydroxy-1,4-dimethyl-1,2,3,4-tetrahydrodibenzo[b,d] furan-1-yl)-6-hydroxybenzofuran-5-yl)ethanone (80), 8-acetyl-1 - (5-acetylbenzofuran-2-yl)- 7-hydroxy-1-methyl-2,3dihydrodibenzo[b,d]furan-4(1H)-one $(81)$, 1-(2-((1S,4S)-8-acetyl-4,7-dihydroxy-1,4-dimethyl-1,2,3,4-tetrahydrodibenzo [b,d] furan-1-yl)-6-hydroxybenzofuran-5-yl)ethanone (82) and seven known benzofuran derivatives (83-89), extracted from the plant Eupatorium chinense showed antiviral activities [31]. Four known benzofurans, (S)-2-methyl-2,3-dihydrobenzofuran-4-carboxylic acid (90), (S)-6-hydroxy-2-methyl-2,3-dihydrobenzofuran-4-carboxylic acid,(91), (S)-5,7-dichloro-6-hydroxy-2-methyl-2,3-dihydrobenzofuran-4-carboxylic acid (92), (S)-5,7-dichloro-6-methoxy2-methyl-2,3-dihydrobenzofuran-4-carboxylic acid (93) isolated from Pinus strobes showed anti-microbial activity [32].
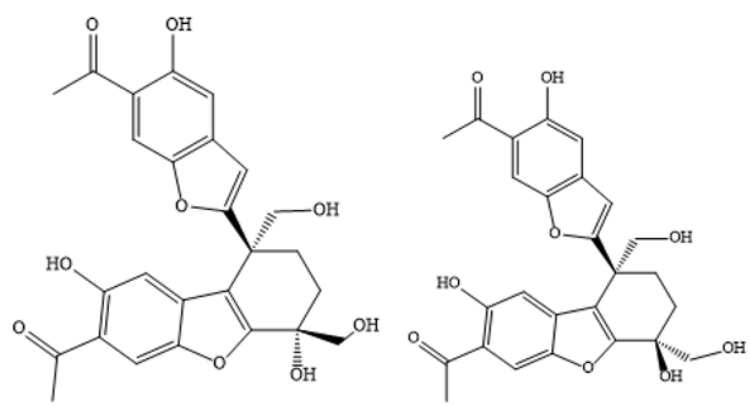

$76(+)-10 \mathrm{R}, 10$ 'S; (-)-10S, 10'R $77(+)-10$ S, 10'S; (-)-10R, 10'R



$78 \mathrm{R}=\mathrm{H}$

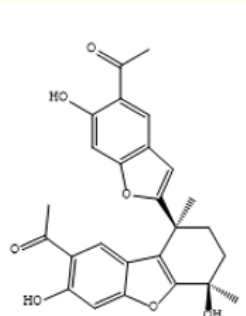

$80(+)-10 R, 10^{\prime} S ;(-)-10 S, 10^{\prime} R$ $81(+)-10 \mathrm{~S}, 10^{\prime} \mathrm{S} ;(-)-10 \mathrm{R}, 10^{\prime} \mathrm{R}$<smiles>[Y4]CC(C)(O)c1cc2cc(C(=O)O)c([R4])cc2o1</smiles>

$R_{1} \quad R_{2}$

$84 \mathrm{H} \quad \mathrm{H}$

$85 \mathrm{OH} \quad \mathrm{H}$

$86 \mathrm{OH} \quad \mathrm{OH}$

$87 \mathrm{H} \quad \mathrm{OH}$

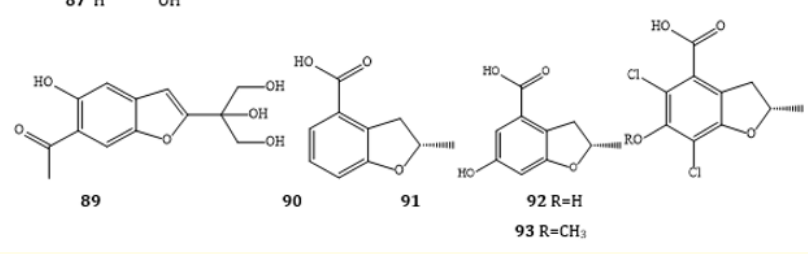

Figure 5

\section{Other activities}

Benzofurans exhibited miscellaneous activities i.e., edible, medicinal, mosquito-repellants, anti-fungal etc. A novel benzofuran-type compound, cathayenone A, i.e., (E)-1-(2,6dihydroxy-4-methoxy-3-phenyl-2,3-dihydrobenzofuran-7-yl)3-phenylprop-2-en-1-one (94), extracted from "Carya cathayensis Sarg" have germicidal effects (anti-fungal effects) on the pathogenic fungi [33]. Four novel compounds (9R)-moracin P 30-0- $\beta$-L-arabinopyranoside (95), (9R)-moracin P 9-0- $\beta$-Dglucopyranoside (96), (9R)-moracin P 30-0- $\beta$-D-glucopyranoside (97) and (9R)-moracin 0 10-0- $\beta$-D-glucopyranoside (98) and ten known compounds, 5-(6-(2-hydroxypropan-2-yl)-5,6dihydrobenzo[1,2-b:5,4-b']difuran-2-yl)benzene-1,3-diol (99), (R)-5-(5-(2,3-dihydroxy-3-methylbutyl)-6-hydroxybenzofuran2-yl)benzene-1,3-diol (100),(R)-5-(6-hydroxy-7,7-dimethyl-6,7dihydro-5H-furo[3,2-g]chromen-2-yl)benzene-1,3-diol (101), mulberroside C (102) 2-(3,5-dihydroxyphenyl)benzofuran-5,6diol (103),4-((3aS,3a1R,8aR,14bS)-12-(3,5-dihydroxyphenyl)6-hydroxy-2-methyl-3a,3a1,8a,14b-tetrahydro-3H-enzo[3,4] isochromeno[1,8-bc]furo[3,2-g]chromen-8a-yl)benzene-1,3-diol (104), (3aS,3a1R,8aR,13bS)-8a-(2,4-dihydroxyphenyl)-6-(6-hy- 
droxybenzofuran-2-yl)-2-methyl-3a,3a1,8a,13b-tetrahydro-1Hbenzo[3,4]isochromeno[1,8-bc]chromene-4,11-diol

(105), (5aR,10aS)-2-((1S,2R,3S)-2-(2,4-dihydroxybenzoyl)-2',4'-dihydroxy-5-methyl-1,2,3,6-tetrahydro-[1,1'-biphenyl]-3-yl)-1,3,8,10atetrahydroxy-5a-(3-methylbut-2-en-1-yl)-5aH-benzofuro[3,2-b] chromen-11(10aH)-one (106), (5aR,10aS)-2-((1S,2R,3R)-2-(2,4dihydroxybenzoyl)-2',4'-dihydroxy-5-methyl-1,2,3,6-tetrahydro[1,1'-biphenyl]-3-yl)-1,3,8,10a-tetrahydroxy-5a-(3-methylbut2-en-1-yl)-5aH-benzofuro[3,2-b]chromen-11(10aH)-one (107), sanggenon D (108) are extracted from the roots of Cortex Mori Radicis showed significant protective activities against glutamate-induced neurotoxicity. Correspondingly, they also exhibited remarkable analgesic activities by inhibition of the acetic acid-induced pain [34]. A new tetrahydrobenzofuran derivative, (6S,7S)-6,7-dihydroxy-3,6-dimethyl-2-isovaleroyl-4,5,6,7-tetrahydrobenzofuran (109) and a known benzofuran derivative, i.e., (6S,7R,7aS)-6, 7-dihydroxy-3,6-dimethyl-5,6,7,7a-tetrahydrobenzofuran-2(4H)-one (110) extracted from an edible mushroom fruit (Lentinus squarrosulus) used as health promoting benefits, and for the research community interested in bioactive compounds for further research [35]. Phenostereum (A), 2-hydroxy-1-((R)2-(prop-1-en-2-yl)-2,3-dihydrobenzofuran-5-yl)propan-1-one (111) and novel phenostereum (B), 2-hydroxy-1-((R)-2-(prop1-en-2-yl)-2,3-dihydrobenzofuran-5-yl)propan-1-one (112), extracted from fungus (Stereum sp.) have larvicidal compounds that can be used to control mosquitoes [36]. Furthermore, benzofuran derived from "Styrax perkinsiae" 5-(2-Propen-1-one)-7methoxy-2-(3,4methylenedioxyphenyl) benzofuran (113), 1"-hydryoxyegonol gentiobioside (114) and six known six compounds, Obassioside B (115), egonol (116), egonol glucoside (117), egonol gentiobioside (118), egonol gentiotrioside (119), and masutakeside (120) showed medicinal value [37]. A novel benzofuran compound, 4-methoxy-3a,7a-dihydrobenzo furan-5-carboxamide (121), extracted from the plant Tephrosia purpurea, revealed antiallergic activity [38]. Similarly, another benzofran derivative, i.e., 4-methoxybenzofuran-5-carboxamide (122) also extracted from the plant of Tephrosia purpurea, revealed anti-allergic activity [39]. Biological screening studies indicated that benzofurans displayed a moderate activity against Staphylococcus aureus, which may be due to the existence of a modified eremophilane skeleton. Compounds (1'S,2'S,5'R)-5-hydroxy-5'-((R)-1-hydroxypropan2-yl)-3-oxo-3H-spiro[benzofuran-2,1'-cyclopentane]-2'-carboxylic acid (123) (1'R,2'S,5'R)-5-hydroxy-5'-(3-hydroxyprop-1-en-2-yl)3-oxo-3H-spiro[benzofuran-2,1'-cyclopentane]-2'-carboxylic acid
(124) exhibited from fruits of "Ganoderma lingzhi", are used for the treatment and prevention of many diseases and acts as antifungal agent. It is an edible, very effective and medicinal fungus [40]. Two novel compounds, 8-acetyl-4,7-dihydroxy-4-methyl-3,4dihydrodibenzo[b,d]furan-1(2H)-one (125), 8-acetyl-4,4',7-trihydroxy-4,5'-dimethyl-3,4-dihydro-2H,3'H-spiro[dibenzo[b,d]furan1,2'-furan]-3'-one (126), isolated from Eupatorium chinense used as folk medicines [41]. Two novel compounds, (6aR,11aR)-2,4dihydroxy-3-methoxy-6a,11a-dihydro-5H-benzofuro[3,2-c]isochromen-5-one (127), (6aR,11aR)-2,4-dihydroxy-1,3-dimethoxy6a,11a-dihydro-5H-benzofuro[3,2-c]isochromen-5-one showed Oestrogenic and anti-platelet activities, which is isolated from Liriope platyphylla [42]. 2-(prop-1-en-2-yl)-2,3-dihydrobenzofuran-5-carbaldehyde (129) isolated from $H$. annosum s.s. and H. irregulare [43], shoreaketone (130)1a and (131) 1b extracted from Dipterocarpaceaeous [44], showed miscellaneous activity. Compound (132), also showed antifungal agents [45]. 2-(2,3-dihydroxy-5-methoxyphenyl)-6-hydroxybenzofuran-3-carbaldehyde (133) and 2-(2,3-dihydroxy-5-methoxyphenyl)-6-methoxybenzofuran-3-carbaldehyde (134) were extracted from S. Grandiflora also exhibited anti-fungal properties [46].


$98 \mathrm{R}=\beta$-D-glucosyl

99

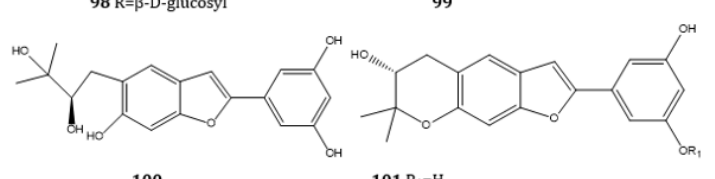

100

$101 \mathrm{R}_{1}=\mathrm{H}$

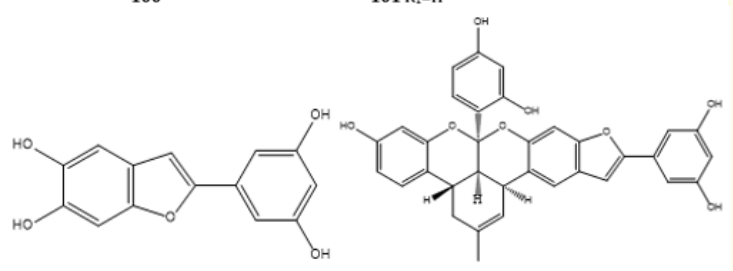

103
104

Citation: Kavita Khatana and Anjali Gupta. "An Update on Natural Occurrence and Biological Activity of Benzofurans". Acta Scientific Medical Sciences 4.10 (2020): 114-123. 


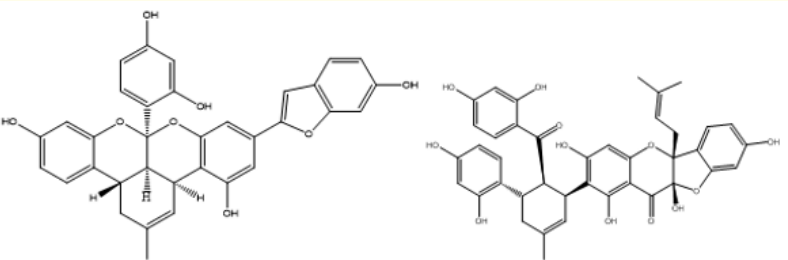

105

106

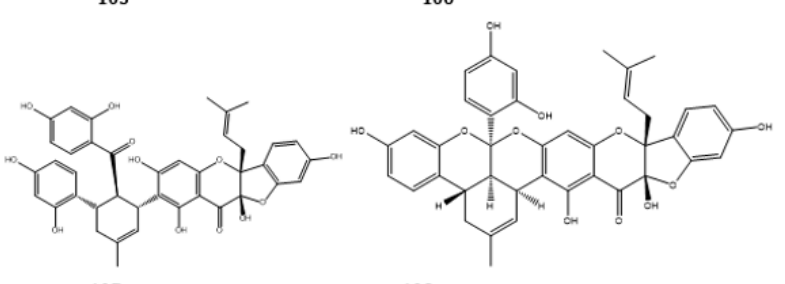

107

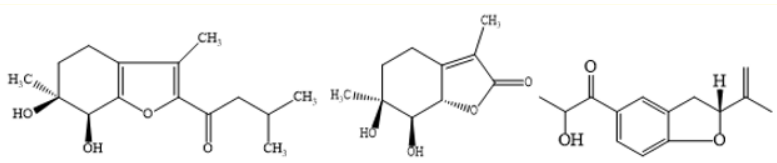

109

110

111<smiles>C=CC(=O)c1cc(O)c2oc(-c3ccc4c(c3)OCC4)cc2c1</smiles>

112



114<smiles>[3H]C(CCO)c1cc(O)c2oc(-c3ccc4c(c3)OCO4)cc2c1</smiles>
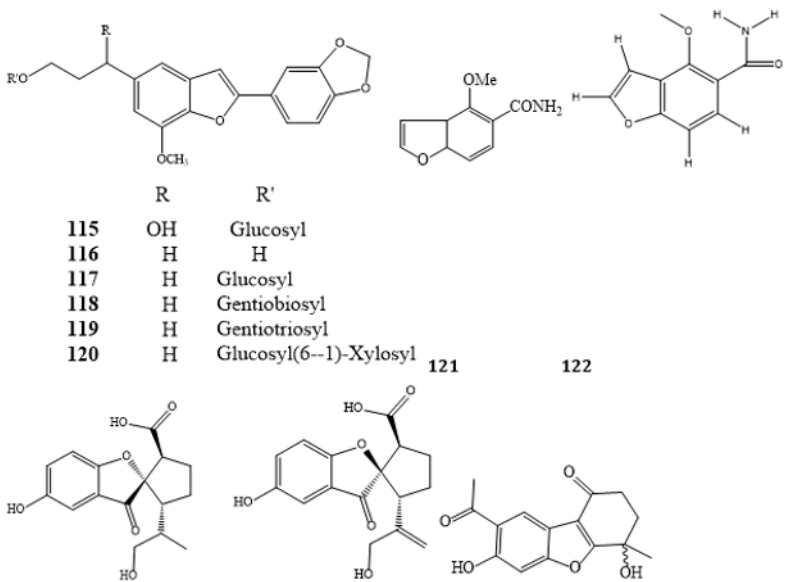

123



$126(-)-10 \mathrm{~S}, 16 \mathrm{~S}$; (+)-10R, 16R
$\mathrm{R}^{\prime}$

Glucosyl

$\mathrm{H}$
Glucosyl

Gentiobiosy

Gentiotriosyl

Glucosyl(6--1)-Xylosyl 121

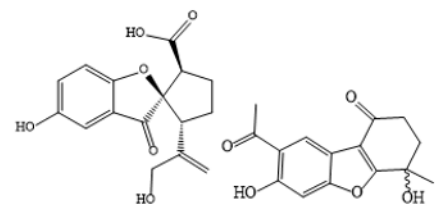

124

125

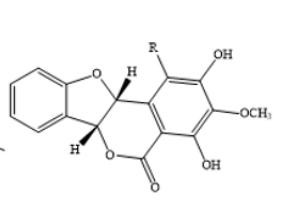

$127 \mathrm{R}=\mathrm{H} 128 \mathrm{R}=\mathrm{OCH}_{3}$

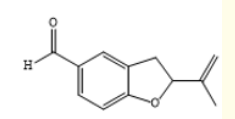

129

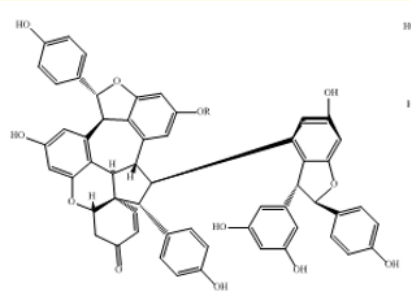

130 Extended rotamer (1a).

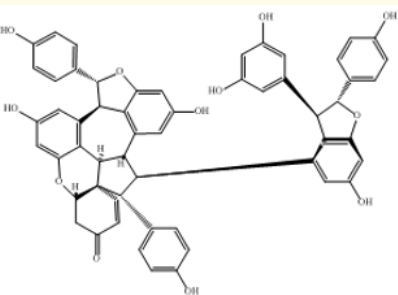

131 Compact rotamer (1b)

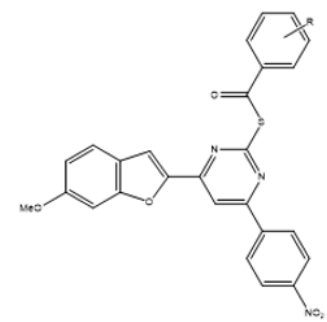

132
$133 \mathrm{R}=\mathrm{H}, 134 \mathrm{R}=\mathrm{OCH}_{3}$

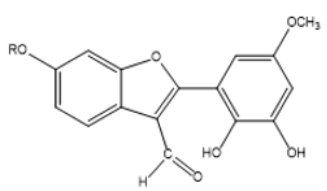

Figure 6

\section{Conclusion}

Benzofurans and its derivatives have enormous potential to find importance in medicinal chemistry and display multiple biological activities. They developed as a cancer chemo-preventive agent and be useful in cancer therapy to sensitize tumor cells. Many efficient clinical drugs are developed by modifying the benzofuran derivatives. They are playing vital role in HIV treatment, and acts as anti-viral, anti-microbial, anti-fungal, anti-allergic activities. They are used as in oestrogenic, anti-platelet activities, and in modulating the regulation of anti-inflammatory activities. They also help in enzyme inhibition and acts as mosquito repellants too. In short, we can say that benzofuran derivatives show tremendous health benefits, which showed numerous scopes in medicinal, pharmaceutical industrial branches.

\section{Conflict of Interest}

None.

\section{Bibliography}

1. Kamal M., et al. "Benzofurans: a new profile of biological activities". International Journal of Medicine and Pharmaceutical Research 1 (2011): 1-15.

2. An FL., et al. "Cytotoxic flavonol-diamide 3+ adducts from the leaves of Aglaia odorata”. Tetrahedron 71.16 (2015): 24502457. 
3. An FL., et al. "Cytotoxic Rocaglate Derivatives from Leaves of Aglaia perviridis". Drug development Molecular Medicine Scientific Reports 6 (2016).

4. Huang XX., et al. "Cytotoxic and antioxidant dihydrobenzofuran neolignans from the seeds of Crataegus pinnatifida". Fitoterapia 91 (2013): 217-23.

5. Hang DTT., et al. "An amorphane sesquiterpene and a benzofuran glucoside from Eupatorium coelestinum". Phytochemistry Letters 4.3 (2013): 377-379.

6. Hu ZF., et al. "Two new benzofuran derivatives with anti-inflammatory activity from Liriope spicata var. prolifera". Fitoterapia 82.2 (2011): 190-192.

7. Zeng X., et al. "Bioactive phenolics from the fruits of Livistona chinensis”. Fitoterapia 83.1 (2012): 104-109.

8. Yang DS., et al. "Cytotoxic prenylated bibenzyls and flavonoids from Macaranga kurzii”. Fitoterapia 99 (2014): 261-266.

9. Wang M., et al. "Diels-Alder adducts with PTP1B inhibition from Morus notabilis". Phytochemistry 109 (2015): 140-146.

10. Khaleghi F., et al. "A new bioactive compound from the roots of Petasites hybridus". Phytochemistry Letters 4.3 (2011): 254258.

11. Kikuchi H., et al. "Novel chlorinated dibenzofurans isolated from the cellular slime mold, Polysphondylium filamentosum and their biological activities". Bioorganic and Medical Chemistry 21.15 (2013): 4628-4633.

12. Chen XZ., et al. "Biphenyls from aerial parts of Ribes takare". Chinese Chemical Letters 25.1 (2014): 127-130.

13. Kaweetripo W., et al. "5-formylfurfuryl esters from Duabanga grandiflora”. Phytochemistry Letters 76 (2012): 78-82.

14. La Clair JJ., et al. "Ganodone, a Bioactive Benzofuran from the Fruiting Bodies of Ganoderma tsugae". Journal of Natural Products 74.10 (2011).

15. Zhuo HX., et al. "Neolignans from Selaginella moellendorffii". Natural Products and Bioprospecting 6 (2016): 161-166.

16. Mansoor TA., et al. "Apoptosis inducing activity of benzophenanthridine-type alkaloids and 2-arylbenzofuran neolignans in HCT116 colon carcinoma cells". Phytomedicine 20.10 (2013): 923-929.

17. Wu B., et al. "New lignan glucosides with tyrosinase inhibitory activities from exocarp of Castanea henryi". Carbohydrate Research 355 (2012): 45-49.
18. Son NT. "A review on the medicinal plant Dalbergia odorifera species: phytochemistry and biological activity". EvidenceBased Complementary and Alternative Medicine (2017).

19. Liu S., et al. "Pestaloficiols Q-S from the plant endophytic fungus Pestalotiopsis fici”. Fitoterapia 85 (2013): 114-118.

20. Marhol P., et al. "Pharmacokinetics of pure silybin diastereoisomers and identification of their metabolites in rat plasma". Journal of Functional Foods 14 (2015): 570-580.

21. Wang Q., et al. "Three new phenolic compounds from the roots of Glycyrrhiza yunnanensis". Fitoterapia 85 (2013)35-40.

22. Luoa G., et al. "3-Formyl-2-arylbenzofurans from the aerial parts of Itea ilicifolia". Phytochemistry Letters 10 (2014): 1922.

23. Li X., et al. "Phenolics from Leontopodium leontopodioides inhibiting nitric oxide production". Fitoterapia 83.5 (2012): 883-887.

24. Wu XF., et al. Journal of Tetrahedron 67.42 (2011): 8155-8159.

25. Yang DS., et al. "A new Geranylated 2-Arylbenzofuran from Morus alba". Chinese Herbal Medicines 7.2 (2015): 191-194.

26. Dang NH., et al. "2-Benzyl-benzofurans from the tubers of Ophiopogon japonicus". Chemistry Central Journal 11 (2017): 15.

27. Luo G., et al. "Novel 2-arylbenzofuran dimers and polyisoprenylated flavanones from Sophora tonkinensis". Fitoterapia 99 (2014): 21-27.

28. Lee DS and Jeong GS. "Arylbenzofuran isolated from Dalbergia odorifera suppresses lipopolysaccharide-induced mouse BV2 microglial cell activation, which protects mouse hippocampal HT22 cells death from neuroinflammation-mediated toxicity". European Journal of Pharmacology (2014): 728.

29. Li YP., et al. "Two new dihydrobenzofuran-type neolignans from Breynia fruiticosa". Phytochemistry Letters 6.2 (2013): 281-285.

30. Liang WJ., et al. "Paeoveitols A-E from Paeonia veitchii". Fitoterapia 106 (2015): 36-40.

31. Wang WJ., et al. "Antiviral benzofurans from Eupatorium chinensis". Phytochemistry 122 (2016): 238-245.

32. Richardson SN., et al. "Antimicrobial dihydrobenzofurans and xanthenes from a foliar endophyte of Pinus strobus". Phytochemistry Letters 117 (2012): 436-443. 
33. Zhang SY., et al. "Novel benzofuran constituent from the husk of Carya cathayensis Sarg”. Phytochemistry Letters 5.3 (2012): 473-475.

34. Wang YN., et al. "Bioactive Benzofuran Derivatives from Cortex Mori Radicis, and Their Neuroprotective and Analgesic Activities Mediated by mGluR1". Molecules 22 (2017): 236.

35. Isaka M., et al. "A tetrahydrobenzofuran derivative from the fermentation broth of Lentinus squarrosulus". Phytochemistry Letters 4.2 (2011): 106-108.

36. Sun R., et al. "Two new benzofuran derivatives from the fungus Stereum sp. YMF1.1684". Phytochemistry Letters 4.3 (2011): 320-322.

37. CHEN QF, et al. "Two new 2-phenylbenzofurans from the bark of Styrax perkinsiae". Chinese Journal of Natural Medicines 10.2 (2012): 92-97.

38. Shill MC., et al. "The isolation and synthesis of a novel benzofuran compound from Tephrosia purpurea and the synthesis of several synthesis of several related derivatives, which suppress histamine $\mathrm{H} 1$ receptor gene expression". Bioorganic and Medicinal Chemistry 23.21 (2015): 6869-6874.

39. Shill MC., et al. "A novel benzofuran, 4-methoxybenzofuran5-carboximide, from Tephrosia purpurea suppressed histamine $\mathrm{H} 1$ receptor gene expression through a protein kinase $\mathrm{C}$ - $\delta$-dependent signaling pathway". International Immunopharmacology 30 (2016): 18-26.

40. Yan YM., et al. "Metabolites from the mushroom Ganoderma lingzhi as stimulators of neural stem cell proliferation". Phytochemistry Letters 114 (2015): 155-162.

41. Wang WJ., et al. "Two pairs of new benzofuran enantiomers with unusual skeletons from Eupatorium chinense". Tetrahedron Letters 54.26 (2014): 3321-3332.

42. Tsai YC., et al. "The oestrogenic and anti-platelet activities of dihydrobenzofuroisocoumarins and homoisoflavonoids from Liriope platyphylla roots". Food chemistry 140 (2013): 305314.

43. Hansson D., et al. "Secondary metabolite comparison of the species within the Heterobasidion annosum s.l. complex". Phytochemistry Letters 108 (2014): 243-251.

44. Ito T., et al. "Absolute structure of shoreaketone: a rotational isomeric resveratrol tetramer in Dipterocarpaceaeous plants". Tetrahedron 68 (2012): 2950-2960.
45. Anbhule SJ and M Vijey A. "A brief review on current antifungal agents". Drug Discovery Today 10.1 (2014).

46. Noviany N., et al. "Sesbagrandiflorain A and B: isolation of two new 2-arylbenzofurans from the stem bark of Sesbania grandiflora". Natural Product Research (2018).

\section{Assets from publication with us}

- Prompt Acknowledgement after receiving the article

- Thorough Double blinded peer review

- Rapid Publication

- Issue of Publication Certificate

- High visibility of your Published work

Website: www.actascientific.com/

Submit Article: www.actascientific.com/submission.php

Email us: editor@actascientific.com

Contact us: +919182824667 